\title{
IS AUTISM A G-ALPHA PROTEIN DEFECT REVERSIBLE WITH NATURAL VITAMIN A?
}

\author{
Mary N. Megson, M.D., F.A.A.P. \\ Developmental Pediatrician \\ Pediatric and Adolescent Ability Center
}

7229 Forest Avenue

Suite 211

Richmond, VA 23226

Fax: 804-673-9195

\begin{abstract}
Autism may be a disorder linked to the disruption of the G-alpha protein, affecting retinoid receptors in the brain. A study of sixty autistic children suggests that autism may be caused by inserting a G-alpha protein defect, the pertussis toxin found in the D.P.T. vaccine, into genetically at-risk children. This toxin separates the G-alpha protein from retinoid receptors. Those most at risk report a family history of at least one parent with a pre-existing G-alpha protein defect, including night blindness, pseudohypoparathyroidism or adenoma of the thyroid or pituitary gland.

Natural Vitamin A may reconnect the retinoid receptors critical for vision, sensory perception, language processing and attention. Autism spectrum disorders have increased from 1 in 10,000 in 1978 to 1 in 300 in some US communities in 1999. Recent evidence indicates that autism is a disorder of the nervous system and the immune system, affecting multiple metabolic pathways.

Autism has been defined by DSM-IV criteria as a childhood behavioral and neurological disorder with onset prior to three years of age. Autistic children and adults have qualitative impairments in social interaction and communication, including either a delay in or complete lack of language development. Furthermore, many people with autism engage in restrictive patterns of behavior including rigid adherence to routines and/or repetitive motor mannerisms such as hand flapping (1).

Autistic spectrum disorders have increased from 1 in 10,000 in 1978 to 1 in 300 is some US communities in 1999 (2). Recent evidence indicates that autism is a disorder of the nervous system and the immune system, and it affects multiple metabolic pathways.

This study of 60 autistic children and their families suggests that inserting a G-alpha protein defect, namely the pertussis toxin in the D.P.T. vaccine, (3) into genetically at-risk children causes autism. This toxin separates the G-alpha protein from retinoid receptors. Those most at risk report a family history of at least one parent with a pre-existing G-alpha protein defect, exhibited in disorders such as night
\end{abstract}


blindness, pseudohypoparathyroidism or adenoma of the thyroid or pituitary gland (4).

This hypothesis asserts that treating these children with natural cis forms of Vitamin A may have the effect of reconnecting the hippocampal retinoid receptor pathways that are critical for vision, sensory perception, language processing and attention (5).

Many of these especially vulnerable children have tissue types of HREs DR 3, DR4, and DR5 (6). These particular tissue types form the tightest bonds with blocked RAR and RXR retinoid receptors (7).

Autism is a true developmental disorder. Many of these children are exposed to wheat at nine months, followed by exposure to the measles antigen at 12 to 15 months (8). The human measles antibody that is produced cross-reacts with intermediate filaments, which are known to be important for maintaining tight junctions and gap junctions between cells, gut mucosal integrity and cell to cell communication $(10)(11)$.

Many of these children, who need natural, unsaturated cis forms of Vitamin A found in sources such as cold water fish like salmon, or cod, liver, kidney, and milk fat, are not getting this in the modern diet. Instead, they are dependent on Vitamin A Palmitate, found in commercial infant formula and low fat milk. Unfortunately, absorption of Vitamin A Palmitate requires an intact gut mucosal microvilli surface at the right $\mathrm{PH}$, in the presence of bile for metabolism (12). However, many of these children already have damaged mucosal surfaces due to unrecognized wheat allergy or intolerances.

\section{The Role of Vaccinations in G-Alpha Protein Defects}

When the live viral measles vaccine is given, it depletes the children of their existing supply of Vitamin A (13), which negatively impacts the retinoid receptors. Natural Vitamin A, in the cis form, is important for activation of $\mathrm{T}$ and $\mathrm{B}$ cells for long-term immune memory to develop (14) and is necessary for natural killer cell function (15). Scrimshaw, et al. (1968) reviewed over 50 studies of infection and nutrition and wrote, "no nutritional deficiency in the animal kingdom is more consistently synergistic with infection than that of Vitamin A" (16).

If artificial Vitamin A Palmitate binds the now free G-alpha protein, it deactivates by $90 \%$ the "off switch" for multiple metabolic pathways, involved in vision and cell growth, and disrupts hormonal regulation and metabolism of lipids, protein and glycogen (17). Measles, mumps and rubella titers are either significantly elevated or negative, in spite of one or two doses of the vaccine given to many of these children. Fish oils contain one retinoid metabolite, alpha 14 hydroxyretroretinol that has a role in T-cell activation, vision and growth of lymphoblasts (18). Further research is needed to understand the complete role of these metabolites in the immune system.

At 18 months of age, when the pertussis toxin is added, as "lymphocytosis proliferating factor," it creates a chronic autoimmune monocytic infiltration of the lamina propia in the gut mucosa (19) and may disconnect the G-alpha protein pathways, leaving some G-alpha modulated pathways unopposed. Consequently, the non-specific branch of the immune system is turned on, and without retinoid switching, cannot be down-regulated. The metabolic consequences could be far-reaching.

These 60 children and their families reveal possible consequences of losing the "off-switch" in G-alpha protein modulated pathways through abnormalities in lipid, glucose and protein metabolism in hormone regulation and in oncogene suppression and autoimmune disorders.

\section{Case Studies}


Our early experience with treatment with natural cis forms of Vitamin A in Cod Liver Oil (CLO) in these autistic children, followed by stimulation of blocked acetylcholine receptors for neurotransmitters affected with a blockage of G-alpha pathways in the cell, is promising. There are dramatic, immediate improvements in language, vision, attention and social interaction in some of these children, as evidenced by the following case reports.

My earliest evidence came from a ten-year-old boy diagnosed with autism by DSM-IV criteria (20). The patient's parents suspect he has been reading since age four but his inability to communicate made this unverifiable. Over an eight-year period of regular visits I had never heard him speak. Standardized IQ tests revealed moderate mental retardation. His mother developed night blindness and hypothyroidism in college and had responded well to Vitamin A and thyroid hormone replacement. The patient's mother's sister was diagnosed in infancy with gluten enteropathy that had improved on a gluten free diet. She has had lifelong dry eyes and is night blind (treated with amber glasses.)

For these and other reasons I started the boy on cod liver oil (5,000 IU of Vitamin A, given in 2500 IU/b.i.d.) and a gluten free diet. After one week, he began to sit farther from the television and to notice paintings on the walls at home. He had always gone out of his way to follow the sidewalk and driveway to meet the school bus. On Vitamin A, he began to run across the grass directly from the front door to the school bus. After three weeks, he was given a single dose of Urocholine, an alpha muscarinic receptor agonist, to increase bile and pancreatic secretions and indirectly stimulate hippocampal retinoid receptors. It has minimal cardiac effect, is FDA approved, has been used safely in children since the 1970's for reflux, and does not cross the blood-brain barrier, unlike secretin (21). It stimulates postsynaptic cell membranes via receptors for acetylcholine, a neurotransmitter in the parasympathetic system.

Thirty minutes after administration of the Urocholine, the patient, who was sitting in a chair, swung his feet over the side, pointed to a glass candy jar on my shelf and said, "May I have the red Jolly Rancher ${ }^{\circledR}$ please?" He had read the label on the candy in the clear jar. These were the first words he had spoken in eight years, and the first proof that he could read. We took him outside and he said, "The leaves, the leaves on the trees are green! I see! I see!" When I asked to take his picture he looked at the camera, smiled and waved. When he left the office I said, "See you later." He asked, "What time?"

In this child's case, after several weeks of treatment with Vitamin A in CLO 3500 IU/day, the Urocholine acted like a switch. When absorbed, he immediately became socially engaged, made excellent eye contact, hugged his mother tightly and said, "I love you so much," looking at her face. At that point we both realized that this child had a blocked pathway. The change in language and social interaction was dramatic and immediate. Yet he reverted to the pre-treatment state of silence when the dose wore off. On lower daily doses of Urocholine (12.5 mg bid) along with the Vitamin A, his language and social interactions have continued to progress, albeit slowly.

I discussed the case with Dr. Bernard Rimland, head of the Autism Research Institute. He called me later to get permission for a mother in Kentucky to call me. She was frantic because her fourteen-week-old infant had stopped making eye contact, began to stare at lights and fans, stopped cooing and laughing and no longer turned to sound after early normal development. The mother reported she was night blind and had irritable bowel syndrome. By mother's report, the infant was weaned and placed on standard formula, which was tolerated well. An audiological evaluation revealed normal auditory brain stem responses and tympanograms. The child went to a pediatric Ophthalmologist, who stated the child was farsighted. The exam was otherwise normal. The doctor was unable to get the infant to track in daylight, but when he placed an amber screen in front of his eyes he would easily track all objects.

I spoke with the child's pediatrician who obtained a Vitamin A level. The value was $26 \mathrm{ug} / \mathrm{dl}$ (normal is 
30-90 ug/dl). I instructed the mother to add $0.85 \mathrm{cc}$ of CLO (Vitamins A/D) of cod liver oil to a bottle that night, and $0.85 \mathrm{cc}$ CLO to a bottle at $11 \mathrm{am}$ the next day. When the baby woke from his nap, he was back to normal, smiling, laughing, turning to sound, and tracking objects. As a developmental pediatrician, I have followed his development. By his mother's reports, his receptive and expressive language, cognition, fine and gross motor skills are all normal for his age of nine months. He has remained on $0.85 \mathrm{cc}$ CLO without significant increase in his vitamin A and D levels. He has had further immunizations without regression.

In both cases the improvement was so dramatic that it seemed we were dealing with a blocked pathway, presumably in the hippocampus or amygdala, with an intact cortex.

\section{Effects of Blocked Retinoid Receptors}

In December 1998, Ron Evans et al., at Cornell, isolated RAR-B and RXR receptors in the hippocampus in mice, which, when blocked, created long-term potentiation and depression of neurotransmission (22). The hippocampal pathways are important in spatial learning and memory. When mice with these blocked receptors were put in a maze, and then the maze was changed, these mice never learned to accommodate for the change. However, both normal mice and blind mice easily learned the new pathway with subsequent trials. Evans reported that these mutations affected cognitive functions such as learning and memory and reports that the mice acted as if "they had significant visual perceptual deficits." (23)

Of note, the hippocampus, on staining and electromagnetic exam, revealed no anatomic abnormalities. Presynaptic and post-synaptic responses were normal (24), so the authors concluded that the changes involved changes in inhibition or potentiation at the cellular level. The authors suggested that lack of retinoid signaling did not affect neuronal development (25).

Six of the autistic children I have tested also have had hypothyroidism. Recently reported was the association of central hypothyroidism when RXR receptors were blocked (26). These RXR receptors are nonspecific members of the superhormone receptor system, and have been identified as calcitonin/secretin, thyroid and retinoid receptors specific for binding with the short carbon chain cis forms of Vitamin A, found in liver, kidney, milk fat and CLO (27).

In the cell membrane, in the hippocampus (28) and retina (29) are G-alpha proteins with RXR and RAR$B$ receptors that potentiate or depress the signal in a given cell (30). Congenital night blindness is caused by a single protein deletion in G-alpha membrane proteins inside the cell attached to the retinoid receptors which traverse the cell wall, leading to decreased potentiation of the signal (29). Normally, the signal is amplified ten million times from stimulation by the time it exits the $G$ protein coil, providing night vision in conditions of very low light. Gi alpha inhibits cAMP synthesis, closes $\mathrm{Ca}+$ channels and opens K+ channels, while Gs alpha has the opposite effect (30). Both stimulatory and inhibitory G-alpha proteins modulate acetylcholine and adrenergic amines, neurotransmitters and chemokines, all affected in autism (31).

\section{Vitamin A and Urocholine}

Autism may be a disorder linked to the disruption of the G-alpha protein and the resulting effects on the retinoid receptors. These cell membrane proteins are coils that modulate sensory input. Cis forms of retinoids may act by replacing these receptors and by easily penetrating the cell membrane for more direct effects on nuclear retinoid pathways. 
Many children treated with Vitamin A in CLO for two months followed by Urocholine show an immediate improvement in their autistic behaviors including improved eye contact, ability to socialize, and increased language use. Many have been able to toilet train easily and have begun to sleep through the night. Postganglionic parasympathetic muscarinic receptors innervate the bowel and bladder through sacral roots, and the pineal gland where melatonin is produced, through fibers from the upper cervical ganglia. This may be why the children are able to improve their sleep cycles and to toilet train on Urocholine and natural Vitamin A.

One of the first improvements noted on Vitamin A in CLO in children is the dis-appearance of the "sideways" glance at people and objects. By doing this, these children with poor rod function are getting their best three dimensional view of the object by directing light through the pupil onto the fovea (32), which is off-center in the retina, the area of the greatest intensity of red and green cones and greatest acuity. Improved eye contact is noted almost immediately in the autistic children on Vitamin A.

\section{$\underline{\text { Importance of Binding Proteins }}$}

Cellular retinaldehyde-binding protein is important in transferring retinal from the photoreceptor to the retinal plasma epithelia. This binding protein is found in the retina and pineal gland. The human genome has sequence similarities with yeast SEC14 protein, which stimulates secretory activity of the Golgi apparatus (33).* These Muller cells are Potassium sinks, which are RXR and RAR-B receptors modulated by G-alpha proteins (34). If depolarization here is not "intensified" because of a G-alpha protein defect, this may decrease the stimulus to the brain from the neural retina. This protein binds only the 11-cis and other di and tri cis isomers of retinaldehyde to form a stable complex with opsin, the forms found in CLO (36).

P19 cells are neuron stem cells, which, in their response to their RXR and RAR receptors being stimulated by retinoic acid, undergo cell differentiation. As these cells differentiate, they express characteristics of epithelial cells. Many syndromes with neurocutaneous markers are associated with autism. These cells, upon differentiation, have a small voltage outward current, but when differentiated have large inward sodium, potassium and CA+ currents. As mature cells they synthesize acetylcholine, not catecholamines (37). Urocholine stimulates alpha muscarinic postganglionic parasympathetic acetylcholine receptors. Affected $\mathrm{G}$ proteins, trimeric guanine nucleotide binding proteins, rely on signals from protons, hormones, odorants, and neurotransmitters and either decrease transmission, causing less effect when stimulated by hormones, or increase transmission (38). Sensory abnormalities seen in these children may be due to a lack of modulation in signal in the cell membrane, but this warrants further study.

\section{Abnormal Lipid Profiles}

Also, there appeared to be a very high incidence of abnormal lipid profiles in the children. These serum values were drawn prior to the administration of Vitamin A in CLO, due to the known effect of retinol and the synthetic retinoids causing hyperlipidemia. Doses of Vitamin A in CLO in our trial are far below minimal supplemental doses required to induce elevations of lipids, especially triglycerides and VLDL. Of note, supplementation with fish oils with eicosapentenoic acid (EPA) and decosahexenoic acid (DHA) has been reported to reduce these lipid levels (39).

\section{Blocked Neurotransmission}

For many of these children, autism represents blocked neurotransmission that can be reconnected. Correcting immunodysfunction and their metabolic disorders will be important for prevention of future 
early heart, endocrine and malignant disorders of endothelial origin.

To quote Alfred Gilman, winner of the Nobel Prize for his discovery of G-alpha proteins, we have been "barbarians at the gate" of cellular function in multiple organ systems (40). These children have been devastated and we have abandoned them and their families from healthcare and rehabilitative services and appropriate educational opportunities.

The far-reaching metabolic consequences may be enormous, with potential links to not only autism, but dyslexia, attention deficit hyperactivity disorder (ADHD), bi-polar disorder, schizophrenia, Chronic Fatigue Syndrome, fibromyalgia, Type II hyperlipidemia, gluten enteropathy, cancer of the mucous secreting glands, and autoimmune disorders including muscular dystrophy and rheumatoid arthritis. SAdenosylmethionine (SAMe), called a supernutrient, is an enzyme-important in acetylcholine synthesis. Loss of gut mucosal integrity would decrease by $85 \%$ gut absorption of CoA, shunting choline into homocysteine production. Increased production of acetylcholine may explain why a continuous dietary source of this nutrient makes people with multiple disorders feel better. Increased serum homocysteine levels have previously been associated with early cardiac disease (41). The incidence of mucoussecreting malignancies in parents and grandparents of the children in the study was 62 cases within 60 families. Cases of adenocarcinoma of the colon alone were seen in 1 in every 4 families when the lifetime risk is approximately 4 percent (42).

The current clinical trial using Vitamin A in CLO vs. placebo in a double blind, cross-over study is necessary prior to a trial using Vitamin A and Urocholine. Data from this trial is important and will have very broad ramifications, including rethinking infant formula composition and timing of immunizations. If this hypothesis is correct, we are one step closer to treatment and prevention of autism.

\section{$\underline{\text { REFERENCES }}$}

1. Wolaich, ML, Felice, M, Drostart, D. The Classification of Child and Adolescent Mental Diagnoses in Primary Care. Elk Grove Village, Ill: American Academy of Pediatrics, 1996: 316317.

2. Rollens, R. Testimony before the United States House Committee on Government Reform, Aug. 3, 1999. By permission.

3. Farvel, Z, Bourne, HR, Iiri, T. The Expanding Spectrum of G Protein Diseases. N Engl J Med 1999; 340: 1014, 1018.

4. Farfel, Z, Bourne, HR, Iiri, T., p. 1013.

5. Chiang, MY, Misner, D, Kemperman, et al. An Essential Role for retinoid receptors RARß and RXR $\alpha$ in long-term potentiation and depression. Neuron, 1998; 21: 1353-1361.

6. Sporn, M, Roberts, A, Goodman, D. The Retinoids: Biology, Chemistry and Medicine. Raven Press, 1994: 331.

7. Sporn, M, et al., 331.

8. Mandell, GL, Bennett, JE, Dolin, R. Principles and Practice of Infectious Diseases, $4^{\text {th }}$ edn. New York: Churchill Livingstone, 1995: 1523.

9. Cohen, A., Bennett, J. Rheumatology and Immunology, $2^{\text {nd }}$ edn. Orlando:Grune and Stratton, 1986: 442.

10. Sporn, M, et al., 636.

11. Fauci, AS. Harrison's Principles of Internal Medicine, $14^{\text {th }}$ edn. New York: McGraw Hill, 1998:510.

12. Sporn, M, et al., 231.

13. Sporn, M, et al., 231.

14. Sporn, M, et al. 536-537. 
15. Sporn, M, et al. 531-532, 535.

16. Sporn, M, et al.,

17. Berman, D, Gilman, A, Mammalian RGS Proteins: Barbarians at the Gate. J Biol Chem. 1998; 273:1269-1272.

18. Wakefield, A, Murch, S, Anthony, A., et al. Ileal Lymphoid-Nodular Hyperplasia, Nonspecific Colitis And Pervasive Developmental Disorders In Children. Lancet 1998; 351:639.

19. Sporn, M, et al. 536-537.

20. Wolraich, ML, 316-317.

21. Arky, R, Physicians Desk Reference, $52^{\text {nd }}$ edn. Montvale, NJ: Medical Economics Co.; 1997:1759.

22. Chiang, et al., 1353-1361.

23. Chiang, et al., 1359.

24. Chiang, et al., 1356.

25. Chiang, et al., 1359.

26. Sherman, SI, Gopal, J, Haugen, BR. Central hypothyroidism associated with Retinoid X Receptor-selective ligands. N Engl J Med 1999; 340:1075-1079.

27. Sporn, M, et al. 333.

28. Chiang, et al., 1353-1361.

29. Sporn, M, et al., 353.

30. Farvel, Z, et al., 1012.

31. Meisenberg, G, Simmons, W. Principles of Biochemistry, St. Louis: Mosby, 1998:577-594.

32. Sporn, M, et al., 352 .

33. Luscher, C, Jan LY, Stoffel, M et al. G Protein Coupled Inwardly Rectifying K+ Channels (Girks) Mediate Postsynaptic But Not Presynaptic Transmitter Actions In Hippocampal Neurons. Neuron 1997; 3:687-695.

34. Zahraoui, A, Touchot N, Chardin, P, Tavitian, A. The Human Rab Genes Encode A Family Of GTP-Binding Proteins Related To Yeast YPT1 And SEC4 Products Involved In Secretion. J Biol Chem 1989; 264:12394-12401.

35. Sporn, M, et al.,356.

36. Sporn, M, et al.,356.

37. Sporn, M, et al., 484-485.

38. Farvel, Z, et al., 1012-1015.

39. Sporn, M, et al., 650 .

40. Berman, DM, et al., 1269-1272.

41. Nygard, O, Nordrehaug, JE, Refsum, Ueland, PM, Farstad, M, Vollset SE. Plasma Homocysteine Levels and Mortality in Patients with Coronary Artery Disease. N Engl J Med 1997; 337:

42. Wijnen, JT, Vasen, HFA, Khan, PM, Zwinderman, AH, et al. Clinical Findings With Implications For Genetic Testing In Families With Clustering Of Colorectal Cancer. N Eng J Med 1998; 339:511. 